FOLIA SCANDINAVICA VOL. 20 POZNAŃ 2016 DOI: $10.1515 / \mathrm{fsp}-2016-0033$

DE DE GRUYTER OPEN

$G$

PRESSto.

\title{
REZEPTIONSÄSTHETISCHE VERWANDLUNG DER MÄRCHEN VON HANS CHRISTIAN ANDERSEN IM 19. UND 20. JAHRHUNDERT IN POLEN
}

\author{
MARIA KRYSZTOFIAK \\ Adam Mickiewicz Univeristy in Poznań
}

ABSTRACT. The paper describes the process of a literary work's perception from the perspective of transformations made in Polish translations of H.C. Andersens's work. The author presents the historical perspective of translating Andersen into Polish in the $19^{\text {th }}$ and $20^{\text {th }}$ century and, based on selected examples, analyzes such essential issues within the realm of artistic translation as translation policy/publishing policy as well as the translator's culture-formative role and tasks. The analyzed issues also include recent and older translations, the culture of translation and, last but not least, the role of translational and literary criticism in the reception process.

\section{ZUR ART DER VERMITTLUNG FREMDSPRACHIGER WERKE}

Der dänische Märchenerzähler Hans Christian Andersen gehört zu denjenigen weltberühmten Schriftstellern, die den Ruhm im Ausland relativ schnell dank vielen Übertragungen in fremde Sprachen erreicht haben. Wenn die Vermittlung eines Autors an die Leser nicht in der Originalsprache stattfindet, sondern mit Hilfe der Übersetzungen und des ganzen Vermittlungssystems, das in jeder Kultur mit differenzierten Institutionen und Mechanismen ausgestattet ist, dann sollten in der literarischen Öffentlichkeit bestimmte Probleme aufgegriffen werden. Eine der wesentlichsten Problemstellungen ist zweifelsohne die Hervorhebung der meistens außer Acht gelassenen Tatsache, dass die Rezeptionsästhetik der fremdsprachigen Literatur die Präsenz eines Kulturtextes und seines Autors zu einem bestimmten Zeitpunkt, je nach literarhistorischen, politischen oder marktbedingten Voraussetzungen, entsprechend konstituieren kann. Der Geschichte der Übersetzungen und der literaturwissenschaftlich, 
komparatistisch und rezeptionsästhetisch orientierten Übersetzungs-theorie ist $\mathrm{zu}$ entnehmen, dass ein für den Verfasser und Übersetzer, aber auch für den Leser, akzeptabler Rezeptionsprozess mehrere Bezüge umfassen soll, vor allem jedoch müssten folgende Überlegungen dem Übersetzer und Verleger bewusst sein:

- Übersetzungen sind, im Sinne Johann Gottfried Herders (2001), Dokumentationen unseres (d.h. des Übersetzers als Repräsentanten seiner Zeit) Verstehens der Originalvorlage

- Das vom Übersetzer gestaltete Bild der Vorlage nimmt seinen Ursprung in den konstitutiven Merkmalen des Originaltextes

- Das vom Übersetzer entfaltete Bild des Originals gilt in der jeweiligen Übersetzungskultur als die Form der Aufbewahrung des fremden Textes für lange Zeit, wenn nicht für immer

- Jede neue Übersetzung verleiht dem gleichen Text ein neues weiteres Sein in der neuen Zeit

- Die Qualität und die Art der Vermittlung des fremden Textes hängt von der Repräsentativität des Übersetzers ab. ${ }^{1}$

\section{AUSGEWÄHLTE EINBLICKE IN DIE POLNISCHE REZEPTION DER MÄRCHEN ANDERSEN IM 19. UND 20. JAHRHUNDERT}

Der in Polen von Anfang an bewunderte und seit 1840 übersetzte Andersen war im 19. und 20. Jahrhundert dem, den dänischen Lesern vertrauten Dichter Andersen eigentlich kaum ähnlich. Als ich Anfang der achtziger Jahre des 20. Jahrhunderts die Ergebnisse meiner vergleichenden übersetzungskritischen Analysen zu veröffentlichen begann (vgl. Krysztofiak, 1982) und als Beweis eine neue, aus dem Dänischen angefertigte Übertragung des Märchens über das hässliche Entlein Den grimme AElling im Verlag Wydawnictwo Śląsk herausgegeben habe (vgl. Andersen, 1985), wurde es von der polnischen Literaturkritik kaum wahrgenommen. Man war noch nicht bereit, die bisher unterschätzte Kultur schaffende Rolle der Übersetzung und der Übersetzungskritik wahrzunehmen und in die öffentliche Kulturdebatte einzubetten.

Die Rezeption der ins Polnische übersetzten Werke von Andersen wurde 1970 von Zdzisława Brzozowska in ihrem Buch über Andersen in Polen sorgfältig dokumentiert (vgl. Brzozowska, 1970). Außerdem entstanden in Polen in den fünfziger und sechziger Jahren des 20. Jahrhunderts zwei monographische Abhandlungen über Hans Christian Andersen, die eine wurde von dem bekannten Literaturhistoriker Zdzisław Żygulski (1956) und die andere von der Übersetzerin aus dem Deutschen Maria Kurecka (1965) verfasst. Beide Monographien geben einen der damaligen Zeit und dem damaligen Forschungsstand entsprechenden Einblick in das Leben und Schaffen des

\footnotetext{
${ }^{1}$ Vgl. Goethe's Werke (1827:237ff., dazu besonders S. 238).
} 
dänischen Schriftstellers, sie enthalten aber keine literarischen bzw. komparatistisch profilierten Textanalysen.

In der Zeit zwischen den vierziger Jahren des 19. Jahrhunderts und Ende des 20. Jahrhunderts erschienen, wie es vor allem der sorgfältig zusammengestellten Bibliographie $^{2}$ der skandinavischen Literatur in Polen zu entnehmen ist, über 400 Auflagen der Werke von Andersen, wobei eine Auflage der Märchen in Polen etwa 50000-100000 Exemplare hatte, auf jeden Fall gilt das für die zweite Hälfte des 20. Jahrhunderts. Mit der Veröffentlichung der Übersetzungen im 19. Jahrhundert befassten sich vor allem Zeitschriften, die in Städten wie Posen, Warschau und Lemberg herausgegeben wurden. Ausgewählte Märchen sind in Buchform erschienen, wie z.B. 1899 in Warschau in dem bekannten und hochgeschätzten Verlag Gebethner\&Wolf, es waren 29 Märchen. Dieser Ausgabe folgten andere Fassungen, u.a. 1902 im Verlag Michał Arct, 1913 und 1931 im Verlag Jakub Mortkowicz. Die letzte Sammlung umfasste 6 Bände und enthielt 140 aus dem Deutschen von Stefania Beylin übertragene Märchen, unter Mitarbeit des Übersetzers Stanisław Sawicki, der als der dänischen Sprache mächtig die Übersetzung von Beylin korrigierte. Vor dem Zweiten Weltkrieg erschienen die Märchen auch in dem Verlag Wydawnictwo Polskie von Rudolf Wegner in Posen, in einer anderen Übersetzung, ebenfalls aus dem Deutschen, nämlich von Franciszek Mirandolla. Außerdem gab es viele Ausgaben der Märchen, die nicht als Übersetzung, sondern als Bearbeitung von Übersetzern vermittelt wurden, zu den Übersetzern, die die Märchen Andersens als Bearbeitung, meistens ebenfalls aus dem Deutschen, auf jeden Fall nie aus dem Dänischen, vorbereitet haben, gehörten u.a. Janina Colonna-Walewska und Cecylia Niewiadomska.

Nach 1945 hat sich der Büchermarkt mit der Übersetzung von Stefania Beylin angefreundet, die für die späteren Ausgaben von dem Schriftsteller und Übersetzer, auch aus dem Dänischen, Jarosław Iwaszkiewicz revidiert wurden, sechs Märchen übersetzte Iwaszkiewicz für die repräsentative Auswahl im Jahre $1975^{3}$. Die Besonderheit dieser Ausgabe bestand außerdem in der Hinzufügung von bisher in Polen nicht veröffentlichten Märchen, wie u.a. Abc-bogen, Psychen, Dryaden, Dandse, dandse Dukke min. Bis Ende des 20. Jahrhunderts waren diese Ausgaben ausschlaggebend für die Lesart und für jegliche Diskussionen über Hans Christian Andersen in Polen.

\section{SIMPLE SPRACHE UND UNÜBERTROFFENE BILDHAFTIGKEIT DER ORIGINALVORLAGE ALS HERAUSFORDERUNG FÜR DIE ÜBERSETZER}

Der dänischen, aber auch der internationalen Andersen-Forschung, insbesondere in der zweiten Hälfte des 20. Jahrhunderts, war zu entnehmen, dass sich die Märchen des Dänen von anderen, darunter auch skandinavischen Märchen, durch ihre

\footnotetext{
${ }^{2} \mathrm{Vgl}$. Andersen (1975).

${ }^{3}$ Vgl. Andersen (1975).
} 
gezielte, manchmal raffinierte, bewusst ästhetisch konstruierte Simplizität der Sprache und konsequente Wiederholbarkeit der stilistisch einfachen Erzählstrukturen abhebt. Mehrmals wurden diese konstitutiven Merkmale sowohl aus der literarhistorischen als auch übersetzerischen Perspektive von Literaturhistorikern, Kritikern und Übersetzern, u.a. von Elias Bredsdorff (1979, hierzu besonders S. 141), Leif Ludwig Albertsen (1972), aber auch von Hanne Marie und Werner Svendsen (1964:258) hervorgehoben, insbesondere in Bezug auf Märchen wie Den grimme Alling, Den lille Havfrue, Sneedronningen, Skyggen, Klokken, Den lille Pige med Svovlstikkerne, die u.a. als ,,weitauslaufende und tiefsinnige Gedankendichtungen“, so Hanne Marie und Werner Svendsen, bekannt sind. $\mathrm{Ob}$ in den polnischen Übersetzungen den genannten Märchen von Andersen die erwähnten Merkmale zu erkennen sind, bleibt dahingestellt. Warum dem so ist, wäre am Beispiel von mehreren Märchen zu beweisen, hier nur einige Hinweise im Zusammenhang mit der Übersetzung von Stefania Beylin des Märchens Den lille Havfrue (Mata syrena). Der Erzähler konstruiert in diesem Märchen die dargestellte Welt aus der Perspektive der Titelfigur, mittels einiger Schlüsselwörter, die die emotionale bzw. innere und reale bzw. äußere Aura sowohl der ganzen Geschichte als auch der Hauptfigur prägen, sie schaffen sonst einen wertvollen Freiraum für die Lesart des Märchens. Es sind solche Universalschlüsselwörter wie u.a. „Længsel“, „Kærlighed“, „Familie“, „Hjem“, „Gud“, sie bilden auch eine wichtige Instanz für die distanzierten und neutral formulierten Kommentare des Erzählers. In den polnischen Ausgaben der Übersetzungen von Stefania Beylin wurde dieser Freiraum, wahrscheinlich unbewusst, dezidiert eingeschränkt. Die Einschränkungen sind vor allem als Resultate einer anderen, katholisch untermauerten Kulturidentität der polnischen Leser und somit auch der Übersetzer zu betrachten. Die in der Übersetzung dieses Märchens vermittelten narrativen Konstruktionen und Kulturbilder machen weder auf die simplen, offenen Erzählstrukturen noch auf die Schlüsselwörter aufmerksam, geschweige denn, dass sie sie wiedergeben. Die folgenden Beispiele bestätigen ernstzunehmende Abweichungen von der Neutralität des Erzählers und von der simplen, auf Universalschlüsselwörter zurückgreifenden Satzkonstruktion. Im Endeffekt wird dem polnischen Leser die Lektüre einer im pathetischen Stil, mit enorm vielen, das Verhalten der Hauptfigur eindeutig erklärenden Hinzufügungen vorgegeben, an Stelle der bildhaft auf Dänisch erzählten Geschichte. Als überzeugendes Beispiel, dass es möglich ist, diese Merkmale ins Auge zu fassen und sie in der Übertragung adäquat wiederzugeben, werden der Originalvorlage und der polnischen Übersetzung entsprechende Zitate aus der englischen Übertragung aus dem Dänischen von Reginald Spink, ebenfalls aus den sechziger Jahren des 20. Jahrhunderts angeschlossen. ${ }^{4}$

\footnotetext{
${ }^{4}$ Dänische Zitate aus den kommentierten Märchen stammen aus: Dal et al. (1963-1990). Zitate in polnischer Sprache stammen aus: Andersen (1975). Zitate in englischer Sprache stammen aus: Andersen (1960).
} 
Dänisch:

Ingen var saa længselsfuld, som den Yngste ... (Bd. 1, S. 89)

Polnisch:

Żadna nie była tak niecierpliwa jak najmłodsza ... (Bd. 1, S. 49)

Englisch:

None was so full of longing as the youngest .... (S. 76)

Das Schlüsselwort „Længsel“, hier als „længselsfuld“ vorgegeben, findet überhaupt keine Widerspieglung in der polnischen Übersetzung, es wurde zu Ungeduld gewandelt.

Das Umfeld der Schlüsselwörter, die mit der Familie eng verbunden sind, wird in der Situation erweitert, in der die Meeresjungfrau erfährt, dass dem Prinzen eine schöne Prinzessin vorgestellt werden soll. So wie die ganze Situation in der Originalvorlage gegen die übliche Vorstellung von den vielfältigen Familienkonventionen, von einer Prinzenfamilie ganz zu schweigen, dargestellt wurde, so sehr fällt die konventional eindeutige Wiedergabe der Situation auf Polnisch auf. Im Original ist noch nicht die Rede vom Heiraten, sondern es geht vor allem um die Freiheit des Individuums in der Familie:

Dänisch:

[...] jeg maa see den smukke Prindsesse, mine Forældre forlanger det, men tvinge mig til at føre hende her hjem, som min Brud, ville de ikke! (Bd.1, S. 103)

Polnisch:

Mam zobaczyć piękną księżniczkę, moi rodzice tego pragną, ale nie zmuszą mnie nigdy, abym ją wprowadził do domu jako moją żonę. (Bd. 1, S. 59)

Englisch:

[...] I must see the beautiful princess; my parents insist on it. But they shall never make me bring her home as my bride [...] (S. 94)

Die Universalschlüsselwörter werden von Andersen stets in ihren lexikalischen Grundformen verwendet, sie werden nicht zu emotionalen Diminutiven umgewandelt, was in den polnischen Übersetzungen sehr oft der Fall gewesen ist, so wie im folgenden Zitat:

Dänisch:

Og den lille Havfrue løftede sine klare Arme op mod Guds Sol, og for første Gang følte hun Taarer. (Bd.1, S. 106)

Polnisch:

Mała syrena wzniosła ku bożemu słonku białe ramiona i po raz pierwszy poczuła w oczach łzy. (Bd.1, S. 61)

Englisch:

And, lifting her clear arms towards God's sun, the little mermaid, for the first time, felt tears. (S. 99) 
Wenn die Sonne Gottes zu göttlichem Sonnlein, so wörtlich, umgewandelt wird, dann sind hier nicht nur keine Schlüsselwörter zu erkennen, sondern darüber hinaus findet eine oberflächliche Ästhetisierung statt, die die ernst und neutral konzipierte Lage verfälscht.

Das nächste Zitat zeigt, wie die Simplizität der Sprachkonstruktion in der polnischen Übersetzung übersehen und zugunsten eines emotionalisierten Ausdrucks geändert wird:

Dänisch:

$\mathrm{Nu}$ gik den lille Havfrue ud af sin Have hen imod de brusende Malstrømme, bag hvilke Hexen boede. (Bd. 1, S. 97)

Polnisch:

I oto mała syrenka porzuciła swój ogródek i poszła w stronę gotującego się wiru, za którym mieszkała czarownica. (Bd. 1, S. 54)

Die englische Übertragung zeigt, dass die adäquate Wiedergabe nicht nur möglich, sondern auch erforderlich ist:

And so the little mermaid set off from her garden for the roaring whirlpools where the witch lived. (S. 87)

Das semantische Umfeld der Universalschlüsselwörter ist in dem Märchen selbstverständlich in einem breiten Kontext der Charaktermerkmale der anderen Figuren sowie der einzelnen Begebenheiten zu sehen. Die lexikalischsemantische Gestaltung dieses Kontexts ist für die Aura der Schlüsselwörter ausschlaggebend und darf im Übersetzungsprozess nicht übersehen werden. Jedes der oben erwähnten Schlüsselwörter ist, trotz ihrer Universalität, selbstverständlich in der Kultur und Sprache der Übersetzung mit differenzierten, manchmal eben recht verschiedenen semantischen Merkmalen ausgestattet. In dem Bedeutungsfeld eines Universalschlüsselwortes werden in jeder Kultur und Sprache aus ihrem Bedeutungsarsenal zusätzlich kulturspezifische, oft sogar entgegengesetzte Bezüge aufgerufen. Die von der polnischen Übersetzerin betonten Bezüge lassen auf ein pathetisches, moralisch und didaktisch eindeutiges und zudem noch recht sentimentales Bild der kleinen Meeresjungfrau, die für ihr neues Glück in der menschlichen Gestalt das vorherige Glück aufopfert, schließen. Wie es aus dem Originaltext und aus den englischen Übersetzungen hervorgeht, treffen diese Merkmale auf die Hauptfigur überhaupt nicht zu, denn in der Sprache von H.C. Andersen erscheint sie als ein von moralisch-didaktischen, emotional gesteuerten Beschreibungen freies Naturwesen, das ein unübertroffenes Interesse für eine andere Welt und eine andere Lebensweise zeigte.

Die bildhafte Simplizität der Sprache von Andersen gehört, wie die Geschichte der Übersetzungen zeigt, zu den größten translatorischen Herausforderungen, die meistens übersehen worden ist. Ein überzeugendes Beispiel 
hierfür finden wir in dem Märchen Sneedronningen (Królowa śniegu), das mit folgenden einführenden Sätzen beginnt:

See saa! Nu begynde vi. (Bd. 1, S. 49)

Die polnische Wiedergabe, auch in anderen Märchen, knüpft an das normalerweise an dieser Stelle erwartete, übliche Appell an die Zuhörer, dass sie sich die Geschichte nun anhören:

Posłuchajcie! Zaczynamy. (Bd. 1, S. 166)

Der dänische Dichter will aber, dass man als Zuhörer sofort auf die Aufnahme der mit der erzählten Geschichte vermittelten Bilder bereit ist.

Es gibt mehrere Gegenstände und ihnen angeschlossenen Ereignisse, die unbedingt als die Auslöser von Bildern im Text ihre Funktion im Vordergrund der Geschichte äußerst prägnant zu erfüllen haben. Man sollte sich in diesem Märchen zum Beispiel den Spiegel vorstellen können, deswegen ist die adäquate Beibehaltung der Erzählperspektive entscheidend, weil es um eine Kette von Ereignissen geht, die direkt mit dem Spiegel, aber nicht mit den, den Spiegel tragenden Kindern zusammenhängen, und gerade das wurde in der polnischen Übertragung nicht eingehalten:

Dänisch:

Jo høiere de fløi med Speilet, des stærkere grinede det, de kunne neppe holde fast paa det; høiere og høiere fløi de, nærmere Gud og Englene; da zittrede Speilet saa frygteligt i sit Griin, at det foer dem du af Hænderne og styrtede ned mod Jorden, hvor det gik i hundrede Millioner, Billioner og endnu flere Stykker, og da just gjorde det megen større Ulykke end før; [...] (Bd. 1, S. 50)

Polnisch:

Im wyżej lecieli z lustrem, tym bardziej wszystko się wykrzywiało, zaledwie mogli je utrzymać, lecieli wyżej, coraz bliżej aniołów i Boga; wtedy lustro zadrżało tak strasznie, że wypadło im z rąk na ziemię, gdzie rozprysło się na tysiąc milionów, bilionów i jeszcze więcej okruchów. Teraz dopiero wyrządzili o wiele większą krzywdę niż przedtem [...] (Bd. 1, S. 166)

Englisch:

The higher they flew with the lokking-glass, the more it grinned, and they could hardly hold on to it. Higher and higher they flew, getting nearer to God and his angels. Then all at once the looking-glass gave such a dreadful twitch while grinning that it shot out of their hands and crashed to the ground, where it broke into hundreds of millions, billions, and even more bits, causing more mischief than before $[\ldots]$ (S. 213)

Die Vorstellung der Kinder von der Märchenwelt und die Art ihres Agierens in Sneedronningen rücken im Märchen in den Vordergrund indirekt durch die Hinzufügung der Psalmzeilen, es sind Zeilen, die bei Andersen zugleich die 
Funktion der Kultursymbole der protestantischen Denkweise erfüllen, d.h. die Kinder finden einen Partner in dem Jesus-Kind für ihr gemeinsames Gespräch. Im katholisch zugeschnitten Kultur-Code wird erwartet und verlangt, dass man sich vor dem Jesus-Kind zunächst mal verbeugt, und nicht unbedingt unmittelbar einredet:

Dänisch:

Roserne voxe i Daler,

Der faae vi Barn Jesus i Tale! (Bd.1, S. 52)

Polnisch:

Róża przekwitła i minie,

Pójdź, pokłońmy się Dziecinie (Bd. 1, S. 168)

Englisch:

The roses grow in the valley,

There we can speak to Jesus (S. 251)

Meine eingehende vergleichende Lektüre der polnischen Übersetzungen der Märchen von H.C. Andersen ließ mich schon damals, in den achtziger Jahren des 20. Jahrhunderts, die Schlussfolgerung von der Notwendigkeit einer aus dem Dänischen vorgenommenen Übersetzung, die alle Märchen von Andersen berücksichtigen würde, an die polnische Öffentlichkeit vermitteln. Es war damals eine Herausforderung an die polnische Literaturkritik, die polnischen Verleger und nicht zuletzt an diejenigen Gremien und Institutionen, die über die Übersetzungspolitik politisch-ideologische Entscheidungen getroffen haben. Ähnliche Forderungen in Bezug auf die Übersetzung der Märchen von Hans Christian Andersen aus der Originalsprache wurden damals auch in anderen Ländern gestellt, in manchen sogar noch früher, dies trifft besonders für Holland und Großbritannien, aber auch Deutschland und die USA zu. In Polen hat man das Problem erst im 21. Jahrhundert ernst genommen, es ist aber erfreulich, dass die von mir 1982 in der in Posen herausgegebenen_Zeitschrift „Nurt“ eingeleitete Debatte ein erfolgreiches Resultat in Form von einer neuen, aus dem Dänischen, von Bogusława Sochańska vorbereiteten Übertragung aller Märchen von Hans Christian Andersen, 2006 erschienen, gebracht hat. Jede neue Übersetzung müsste immer ein Kulturereignis sein und einen Beitrag zur Modellierung der Übersetzungskultur leisten (vgl. Krysztofiak, 2013). Die Übertragung von Bogusława Sochańska kommt diesen Anforderungen weitgehend entgegen und bietet somit auch eine neue Rezeption der Märchen von Hans Christian Andersen (vgl. Krysztofiak, 2012, hier besonders S. 39-46 und 205-214).

Wie schwierig es ist, Texte, Welt- und Kulturbilder, Kultursymbole und Kulturwörter, sowie ästhetische Konstruktionen in eine andere Sprache und Kultur zu transferieren, war aber den Literaturkritikern und Übersetzern in Europa schon sehr früh, auch zu Andersens Zeit bewusst. 
Es ist nichts schwerer, als das Denken eines anderen bis in die feinere Eigentümlichkeit seines Ganzen nachkonstruieren, wahrnehmen und charakterisieren zu können [...]. Und doch kann man nur dann sagen, daß man ein Werk, einen Geist verstehe, wenn man den Gang und Gliederbau nachkonstruieren kann. Dieses gründliche Verstehen nun, welches, wenn es in bestimmten Worten ausgedrückt wird, Charakterisieren heißt, ist das eigentliche Geschäft und innere Wesen der Kritik. ${ }^{5}$

Diese für Übersetzer und Kritiker aufschlussreiche Überlegung galt nicht nur Anfang des 19. Jahrhunderts, als sie von Friedrich Schlegel formuliert wurde, sondern sie dürfte als Wegweiser von immer jüngeren Übersetzern und Kritikern ernstgenommen werden.

Am Ende des Überblicks über die polnische Übersetzungsrezeption von H.C. Andersen sollte man noch eine allgemeine Überlegung zur Kultur der Übersetzung hinzufügen. Durch die Übersetzung werden sowohl Kulturbilder als auch Weltbilder sprachlich transferiert, in beiden Fällen geht es um Phänomene, die zeitlich und räumlich bedingt sind, der Originaltext bleibt nach wie vor unverändert und vermittelt ununterbrochen die gleichen Kulturinformationen, die gleiche Weltanschauung und die gleichen Wertmaßstäbe, die Übersetzung wird immer nur als Ausdruck ihrer Zeit, in der sie angefertigt wurde, wahrgenommen. In der Übersetzung verwandeln sich zeitbedingt die Konstanten des Originals, in der Übertragung werden diese Konstanten vom Übersetzer durch die Umstände moderiert, beispielsweise, wie das mit den polnischen Märchen Andersens schon im 19. Jahrhundert passiert, wird aus dem protestantischen Prätext ein katholischer Zieltext laboriert, außerdem ändert sich die Stilistik der Narration, die schlichte Volkserzählung wird ästhetisch in ein biedermeierlich geziertes Kunstmärchen umgewandelt. Durch etliche Neuauflagen hindurch werden Andersens Märchen stilistisch kaum verändert, von Generation zu Generation schon längst als polnische Kulturtexte gelesen. Die neue Übersetzung im 21. Jahrhundert, erstmalig ganz aus dem Original übertragen, ist zwar semantisch einwandfrei, sie steht aber gegen die Lesetradition in Polen, da sie, objektiv gesehen, andere Emotionen die von der Wortkultur ausgehen, vermittelt.

Als Vorwarnung für die unreflektierte Lesart der Übersetzungen, übrigens nicht nur der polnischen, gilt die Behauptung, vom Übersetzer als dem zweiten Autor des Textes, im Ausland liest man notgedrungen immer nur die Fassung des Übersetzers. Es gibt Ausnahmen, aber selten kommt es vor, dass das dichterische Talent des Übersetzers mit dem Talent des Autors gleichzusetzen ist, und obwohl beide als Schriftsteller anzusehen sind, wirken sie unterschiedlich. Wenn die Übersetzung sich besser als das Original liest, ist sie oft ein hervorragendes Kunstwerk, aber eine falsche Übersetzung, das kommt manchmal auch bei den Übersetzungen der Märchen Andersens vor, und das nicht nur in Polen. Die

\footnotetext{
${ }^{5}$ Schlegel (1988, Bd. 3:50).
} 
Einfachheit der Erzählweise von Andersen strahlt eine besondere zauberhafte Aura aus, eine übermäßige Ästhetisierung zerstört diese Aura. Das Geheimnis der Übersetzungskunst liegt also woanders.

\title{
PRIMÄRLITERATUR
}

Andersen, H.C. (1899). Baśnie. Opracowane przez C. Niewiadomską. Warszawa: Gebethner i Wolff. Andersen, H.C. (1931). Baśnie.[Übersetzt von Stefania Beylin, Stanisaw Sawicki]. T.I-VI. Warszawa: Jakub Mortkowicz.

Andersen, H.C. (1960). Fairy Tales and Stories. [Übersetzt von Reginald Spink]. London: J.M. Dent\&Sons.

Andersen, H.C. (1975). Baśnie. [Übersetzt von Stefania Beylin und Jarosław Iwaszkiewicz]. T.I-III. Warszawa: PIW.

Andersen, H.C. (1985). Brzydkie kaczatko. [Übersetzt von Maria Krysztofiak]. Katowice: Wydawnictwo Śląsk.

Andersen, H,C. (2006). Baśnie. [Übersetzt von Bogusława Sochańska]. T. I-III. Poznań: Media Rodzina.

Dal, E. et al. (Hrsg.) (1963-1990). H.C. Andersens Eventyr 1-7. København: Dansk Sprog- og Litteraturselskab.

\section{SEKUNDÄRLITERATUR}

Albertsen, L. L. (1972). Litterar oversattelse. Vanskeligheder ved gengivelse af fremmede sprogs kunstprosa. København: Gyldendal.

Benjamin, W. (1980). Gesammelte Schriften. Band IV.1. Hrsg. Von Tilman Rexroth. Frankfurt am Main: Suhrkamp.

Bredsdorff, E. (1979). H.C. Andersen. Mennesket og digteren. København: Gyldendal.

Brzozowska, Z. (1970). Andersen w Polsce. Wrocław: Ossolineum.

Goethe's Werke. Vollständige Ausgabe letzter Hand. Fünfter Band West-östlicher Divan. (1827). Stuttgart, Tübingen: J.G. Cotta'sche Buchhandlung.

Herder, J.H. (2001). Abhandlung über den Ursprung der Sprache. Stuttgart: Reclam Verlag. Krysztofiak, M. (1982). O polskich przekładach baśni H.C. Andersena. Nurt 4/1982, S. 26-28. Krysztofiak, M. (Hrsg.). (2012). Transkulturelle Identität und Übersetzungsmodelle skandinavischer Literatur. Frankfurt am Main: Peter Lang Verlag.

Krysztofiak, M. (2013). Einfuihrung in die Übersetzungskultur. Frankfurt am Main: Peter Lang Verlag. Kurecka, M. (1965). Jan Chrystian Andersen. Warszawa: PIW.

Schlegel, F. (1988). Lessings Gedanken und Meinungen. In: E. Behler, H. Eichner (Hrsg.), Kritische Schriften und Fragmente. (Studienausgabe in 6 Bänden). Paderborn: Schöningh.

Svendsen, H. M., Svendsen, W. (1964). Geschichte der dänischen Literatur. [Ins Deutsche übertragen von George Goetz]. Neumünster: Kurt Wacholtz.

Żygulski, Z. (1956). Jan Chrystian Andersen bajkopisarz duński. Warszawa: Wiedza Powszechna.

\author{
Maria Krysztofiak \\ Uniwersytet im. Adama Mickiewicza w Poznaniu \\ Katedra Skandynawistyki \\ al. Niepodległości 4 \\ 61-874 Poznań \\ Poland \\ kry@amu.edu.pl
}

